\title{
INFLUENCE OF THE TOPOLOGICAL STRUCTURES OF THE NOSE OF HIGH-SPEED MAGLEV TRAIN ON AERODYNAMIC PERFORMANCES
}

\author{
Yeteng Wang ${ }^{1,2}$, Zhenxu Sun $^{1,2}$ \\ ${ }^{1}$ Key laboratory for mechanics in fluid solid coupling systems, institute of mechanics, \\ Chinese Academy of Sciences, Beijing, China \\ 'University of Chinese Academy of Sciences, UCAS, Beijing, China
}

\begin{abstract}
In the past few years, considerable attention has been paid to high-speed maglev train in the field of rail transit. The design speed of the high-speed maglev train is $600 \mathrm{~km} / \mathrm{h}$, which is significantly higher than that of the high-speed train. With the increase in operating speed, high-speed maglev trains have higher requirements for aerodynamic shape. Superior performance, the beautiful aerodynamic shape is an important direction for the development of high-speed maglev trains. Based on the Vehicle Modeling Function (VMF) method, the current research has developed a parametric shape design method suitable for the aerodynamic shape of the maglev train's nose. This method can obtain different topological structures of the high-speed maglev train's nose. The current research uses this method to generate four maglev train noses with large appearance differences and uses these train noses to construct four simplified high-speed maglev models. Then this study numerically analyzes the flow fields of different train models and compares the differences in aerodynamic performance including aerodynamic drag, aerodynamic lift and wake characteristics. The $Q$-criterion is used to study the vortex structure and mechanism of different train wake regions, and the vortex propagation process is studied by turbulence kinetic energy (TKE). Studying the difference in the aerodynamic force of different topological shapes will help to improve the aerodynamic performance of high-speed maglev trains.

Keywords: maglev train; high-speed; topological structures; VMF parameterization method; aerodynamic performance; wake characteristics
\end{abstract}

\section{INTRODUCTION}

The birth of maglev train technology origins from the human pursuit of ground traffic speed. The traditional wheel-rail type railway uses the adhesion force between the wheels and the rail to generate thrust for the train. The adhesion coefficient of the train decreases as the train speed increases. The resistance increases rapidly with the increase of train speed. When the train's adhesion coefficient curve and drag coefficient curve intersect, the speed limit of the wheel-rail train is reached. To surpass this speed limit, it is necessary to develop a transportation system that does not rely on wheel-rail contact. Maglev train is a kind of train driven by magnetic levitation force. It uses electromagnetic force to achieve non-contact levitation and guidance between the train and the track. Germany, Japan, Britain, China and other countries have developed magnetic levitation technology. Germany and Japan are in a leading position in the development of magnetic technology. [1] The development of high-speed maglev trains will help improve the national three-dimensional passenger transportation network and stimulate regional economic and technological progress.

It is reported from Raghuathan et al. [2] that with the substantial increase in train speed, air resistance has become the main component of the total resistance of the train, and the aerodynamic characteristics of the train have also become an important factor restricting train speed. At the same time, the aerodynamic problems seriously affect the safety and comfort of the train operation, so it is necessary to pay attention to the aerodynamics of high-speed maglev trains. In the field of train aerodynamics, wheel-rail high-speed trains have received more attention due to their extensive engineering applications, while there are fewer studies on high-speed maglev trains. Numerical method is an important means to analyze train aerodynamics. Compared with traditional experimental methods, numerical simulation is more efficient, flexible and low-cost. WU et al. [3] used the German TR-08 maglev train as a prototype to perform numerical simulations to analyze the air resistance and flow field distribution of the train. ZHOU et al. [4] designed three maglev train heads based on TR-08 and numerically simulated the flow field around the trains which run at $430 \mathrm{~km} / \mathrm{h}$, and obtained the drag coefficients and lift coefficients of the three heads trains, as well as the pressure wave at the intersection. It is concluded that the flat head type has a better aerodynamic performance. LIANG et al. [5] used dynamic grid technology to 
analyze the cross-wind aerodynamic performance of maglev trains under different wind speeds and wind directions. SHU et al. [6] obtained four new head trains by changing the height and length of the longitudinal section of the TR-08 train head. SHU performed numerical simulations on the flow field around the train and compared the aerodynamic drag performance of various head trains. It is concluded that increasing the length can reduce the aerodynamic drag. ZHOU et al. [7] numerically simulated the surrounding flow field of a $600 \mathrm{~km} / \mathrm{h}$ high-speed maglev train to study its aerodynamic load performance and wake structure. MENG et al. [8] studied the influence of the track gap on the aerodynamic characteristics of the maglev train by numerically simulating the two-group maglev train model, and found that the track gap only has an impact on the pressure distribution at the bottom of the train, which affects the aerodynamic lift of the train.

To sum up, most of the previous researches focused on the aerodynamic characteristics and the flow field structure of existing train shapes. A small amount of research involves the active design of the maglev train's nose, but it is also limited to changing the length, height or width on the basis of the existing one to obtain a new one. This method has certain limitations and it is difficult to obtain a new train's nose with huge differences in appearance. The current study develops a parametric modeling method based on mathematical functions for the head of a high-speed maglev train, and selects four heads with large differences in appearance to form four simplified three-group model. The three-group model includes a head car, a middle car and a tail car. Then, three-dimensional steady numerical simulations have been is performed to compare and analyze the aerodynamic characteristics and the flow field structure of the four train models. This paper introduces a parameterization method of train head shape, details of numerical simulation, comparison of four train models' aerodynamic characteristics and wake structure.

\section{PARAMETRIC MODELING \\ 2.1 VMF Parameterization Method}

For the shape parameterization method based on mathematical analysis, Patrice $\mathrm{C}$ et al. [9] once divided it into two categories according to the geometric meaning of the parameters: one is to directly construct the geometric shape, which is suitable for the design without initial shape; the other is to adjust the geometric shape, which is suitable for the design of the existing initial shape. This paper starts with the Vehicle Modeling Function (VMF) method which is one of the direct construction geometry methods to complete the parametric modeling of the high-speed maglev train's nose.

Constructing the NURBS curve is a commonly used parameterization method, but this method has too many design variables and is inefficient in the 3D shape optimization problem. To overcome the shortcomings of NURBS, J.H.Rho et al. [10] proposed a VMF parameterization method for car shape design. The VMF method deforms the Bernstein polynomial, and strengthens the description of the small curvature radius profile. Compared with the NURBS curve, VMF is insufficient in describing complex curves, but can be used to control simpler curves and surfaces because of fewer design variables, it can effectively improve the efficiency of car shape design and optimization. Such design ideas have also been introduced into the research on the head-type optimization design of high-speed trains. Yo-Cheon $\mathrm{Ku}$ et al. [11] took the lead in using the VMF parameterization method in the optimization design of high-speed trains, and extracted four design variables to describe the geometry of the train head. The idea of applying the VMF parameterization method to the optimization design of high-speed trains can provide a reference for the study of the streamline parameterization design of the maglev train in this paper.

The main idea of the VMF method is to select the key geometry profiles of the shape, and generate the key boundary of the research object by constructing mathematical expressions for the geometry lines; generate the curved surface by linear interpolation between the geometry profiles. The parameterized expression of the two-dimensional profile in the VMF method proposed by J.H.Rho et al. is:

$f\left(\frac{x}{c}\right)=\left(\frac{x}{c}\right)^{A 1}\left(1-\frac{x}{c}\right)^{A 2} S\left(\frac{x}{c}\right)+G\left(\frac{x}{c}\right)$

In the formula, A1 and A2 respectively control the curvature of the front end and the back end of the profile. $Y_{1}$ and $Y_{2}$ are the height coordinates of the starting point and ending point respectively; the value range of $\mathrm{x}$ is $[0, \mathrm{c}] ; \mathrm{S}$ is a function of $\mathrm{x}$, and different types of profile lines can be obtained by changing S. By changing A1, A2 and S, a richly shaped two-dimensional profile can be obtained, as shown in Figure 1.

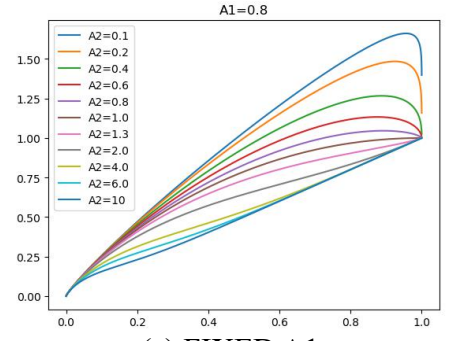

(a) FIXED A1

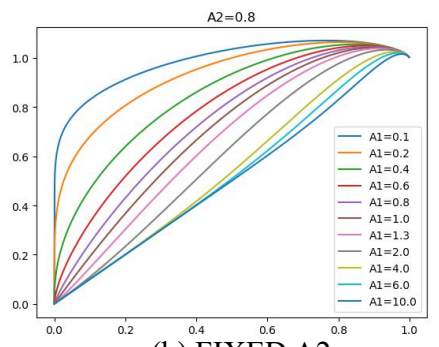

(b) FIXED A2
FIGURE 1: VMF PARAMETRIC METHOD TO GENERATE TWO-DIMENSIONAL CURVE.

\subsection{Construction Topology of Nose}

There are many broken curves and inflection points in the cross-sectional contour of the maglev train. In order to facilitate the surface construction, the maximum cross-section is divided into seven-segment curves in this paper, as shown in Figure 2. The current study keeps the cross-sectional shape unchanged to match the existing carriages. According to the division of the largest cross-section, the basic shape of the maglev train's nose is divided into 7 curved surface blocks, and the boundary of the curved surface blocks is used as the key line of parametric 
modeling, as shown in Figure 3.

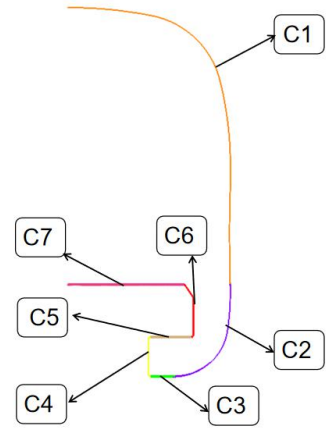

FIGURE 2: CROSS SECTION DIVISION.

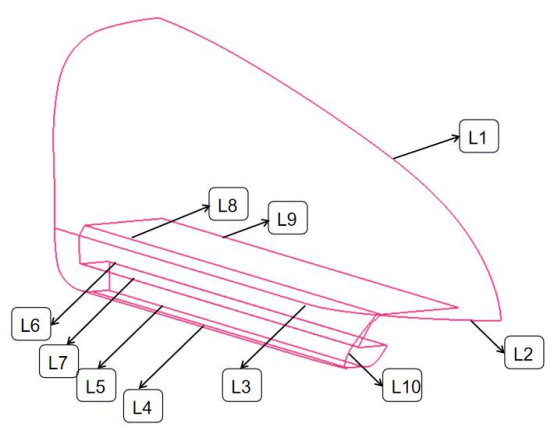

FIGURE 3: KEY LINE SELECTION.

The vertical profile line $\mathrm{L} 1$ and the horizontal profile line L3 are controlled by the improved VMF two-dimensional profile expression (2). The remaining key profile lines are controlled by the start and end point coordinates.

$f\left(\frac{x}{c}\right)=\left(\frac{x}{c}\right)^{A 1}\left(1-\frac{x}{c}\right)^{A 2} S\left(\frac{x}{c}\right)+G\left(\frac{x}{c}\right)$

The specific expression of longitudinal profile line L1 is:

$\left\{\begin{array}{c}z(x)=\left(\frac{x-x_{11}}{x_{12}-x_{11}}\right)^{A_{11}}\left(1-\frac{x-x_{11}}{x_{12}-x_{11}}\right)^{A_{12}} a_{k 1}\left(1-\frac{x-x_{11}}{x_{12}-x_{11}}\right)^{a_{b 1}}+g(x) \\ g(x)=2\left(z_{12}-z_{11}\right)\left(\frac{x-x_{11}}{x_{12}-x_{11}}\right)-\left(z_{12}-z_{11}\right)\left(\frac{x-x_{11}}{x_{12}-x_{11}}\right)^{2}\end{array}\right.$
In the expression, $\mathrm{g}(\mathrm{x})$ is used to control the height and curvature of the endpoint; $x_{11}$ and $x_{12}$ are the $\mathrm{x}$-coordinates of the starting and ending points of the curve; $z_{11}$ and $z_{12}$ are the $\mathrm{z}$-coordinates of the starting and ending points of the curve.

The expression of the horizontal profile line L3 is similar to that of the longitudinal profile, as shown below:

$\left\{\begin{array}{c}y(x)=\left(\frac{x-x_{21}}{x_{22}-x_{21}}\right)^{A_{21}}\left(1-\frac{x-x_{21}}{x_{22}-x_{21}}\right)^{A_{22}} a_{k 2}\left(1-\frac{x-x_{21}}{x_{22}-x_{21}}\right)^{a_{b 2}}+g(x) \\ g(x)=3\left(y_{22}-y_{21}\right)\left(\frac{x-x_{21}}{x_{22}-x_{21}}\right)^{2}-2\left(y_{22}-y_{21}\right)\left(\frac{x-x_{21}}{x_{22}-x_{21}}\right)^{3}\end{array}\right.$

In the expression, $\mathrm{g}(\mathrm{x})$ is used to control the height and curvature of the end point; $x_{21}$ and $x_{22}$ are the $\mathrm{x}$ coordinates of the starting and ending points of the curve; $y_{21}$ and $y_{22}$ are the $y$ coordinates of the starting and ending points of the curve.

Taking the key profile as the skeleton, the basic topological structure of the high-speed maglev train head can be fitted by linear interpolation, as shown in Figure 4. By increasing the $\mathrm{y}$-coordinate value increment, adding a drainage area to the nose cone area can obtain the complete shape of the locomotive, as shown in Figure 5.

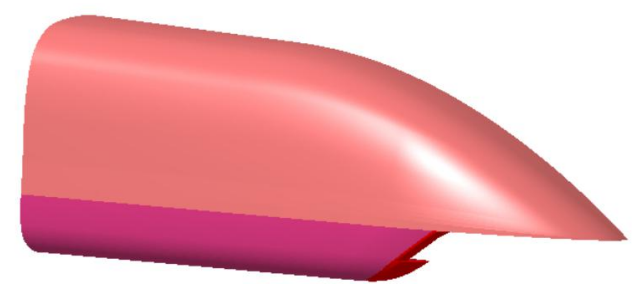

FIGURE 4: BASIC TOPOLOGY.

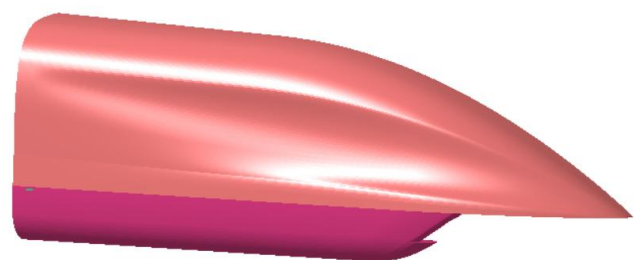

FIGURE 5: COMPLETE TOPOLOGY.

According to the influence of each parameter on the aerodynamic performance and its ability to control the shape, an appropriate number of key parameters are extracted as design variables. The design variables are shown in Table 1.

TABLE 1: DESIGN VARIABLE.

\begin{tabular}{cccccc}
\hline Deformed area & \multicolumn{5}{c}{ Design variable } \\
\hline Profile line L1 & $x_{11}$ & $z_{11}$ & $A_{11}$ & $a_{k 1}$ & $a_{b 1}$ \\
Profile line L3 & $x_{22}$ & - & $A_{21}$ & $a_{k 2}$ & $a_{b 2}$ \\
Starting point of L4 & $x_{41}$ & $y_{41}$ & - & - & - \\
S2 boundary curvature & $\mathrm{smn} 1$ & $\mathrm{smn2}$ & - & - & - \\
Drainage area shape & $h_{n \max }$ & $\mathrm{nns}$ & - & - & - \\
Location of drainage area & $x_{n \operatorname{mix}}$ & $x_{n \max }$ & $z_{n \min }$ & $z_{n \max }$ & - \\
\hline
\end{tabular}




\subsection{Representative Topologies}

The current parametric modeling method can generate a variety of high-speed maglev train head with different topological structures and large differences in appearance. Controlling the longitudinal profile line L1 and the horizontal profile line L2 can deform the basic shape of the head; Controlling the starting point of the bottom surface contour L4 of the train can change the relative position of the bottom surface and the upper streamlined surface; Controlling the drainage area can further control the shape of the curved surface. The parametric modeling method developed in this paper can generate the train head shape with great shape difference and different styles. In this paper, four representative head types are selected from the aerodynamic point of view, named as type I, type II, type III and type IV, as shown in Figure 6. The first is slender head type, the second is flat wide head type, the third is blunt head type, and the fourth is short head type. The study of these four head types can explore the influence of longitudinal section, transverse section shape and nose length on the aerodynamic performance and surrounding flow field of high-speed maglev train.

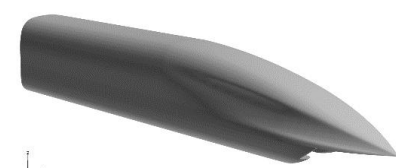

(a)

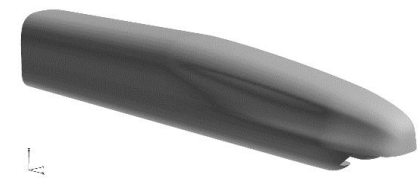

(c)

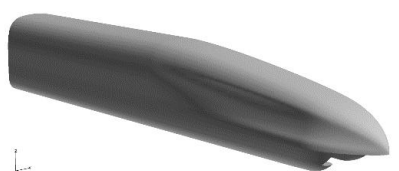

(b)

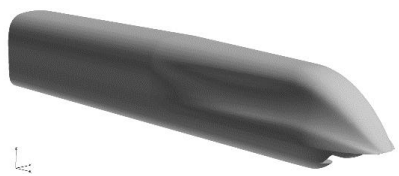

(d)
FIGURE 6: FOUR REPRESENTATIVE HEAD SHAPES:(a) TYPE I; (b) TYPE II; (c) TYPE III; (d) TYPE IV.

The streamline length of the Type I model's head is $12 \mathrm{~m}$. Its longitudinal profile and horizontal profile have the smallest curvature among the four models, presenting a tapered shuttle shape. The streamline length of the Type II model's head is $12 \mathrm{~m}$. The curvature of the longitudinal profile and the horizontal profile is increased compared with the Type I, and the bottom surface is closer to the nose tip, and the contour line is more rounded. The streamline length of the Type III model's head is $12 \mathrm{~m}$. Its longitudinal profile and cross profile curvature are the largest among the four models. The volume is larger than that of Type I and Type II. The streamline shape of the Type III model's nose is close to a bluff body. The shape of the Type IV model's

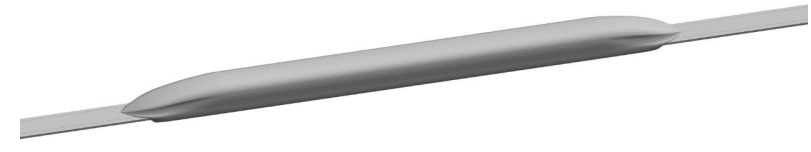

(a) head refers to the urban maglev train. The streamline of the nose is shortened to $5 \mathrm{~m}$, and the streamline is relatively passivated.

In the subsequent chapters of this paper, a simplified three-group model is established with four head types for numerical simulation, and the aerodynamic characteristics and the flow field structure of the four train models are analyzed and compared. Discussion on the four different head types can provide reference for the head type selection and further optimization of high-speed maglev train.

\section{NUMERICAL METHODOLOGY and VALIDATION 3.1 Computational Method}

In this study, the aerodynamic characteristics and the flow field structure of the train are explored under the conditions of open line, no crosswind and smooth operation of the train. In this case, the relative motion between the train and the ground and air is simulated by keeping the train still and giving the incoming flow and boundary velocity.

Numerical simulation has been adopted in various industrial fields $[12,13,14]$, and is one of the main research methods for high-speed train aerodynamics, which owns the advantages of low cost and high efficiency. Numerical solutions can be achieved by commercial software Star $\mathrm{CCM}+$. Because the incoming flow Mach number is about 0.5, the air compressibility needs to be considered. The governing equation is a three-dimensional steady and compressible N-S equation. The discrete method uses the finite volume method based on the cell center, and the spatial discrete format uses the Roe format, and the time discrete uses the LU-SGS implicit discrete method. The turbulence model is the SST $\mathrm{k}-\omega$ model. Li et al. [15] compared different RANS turbulence models to evaluate aerodynamic performance of trains in crosswind, and found that the most accurate model for predicting train surface pressure is the SST k- $\omega$ model. Considering the influence of the boundary layer effect, prism layer grids are generated on the train's surface of the train and strips, which keeps the value of $y+$ of the layer near the train surface around 1.

\subsection{Computational Models}

The main research object of this study is the topological structure of the maglev train's nose. In order to improve the computational efficiency, this paper appropriately simplifies the train model. The shape and aerodynamic characteristics of the middle compartments of the high-speed maglev train are basically the same. Therefore, the calculation model of this study is a simplified three-group structure consisting of a head, a tail and a middle compartment, without windshields and air conditioning boxes. Simplified three-group models for four trains are shown in Figure 7.

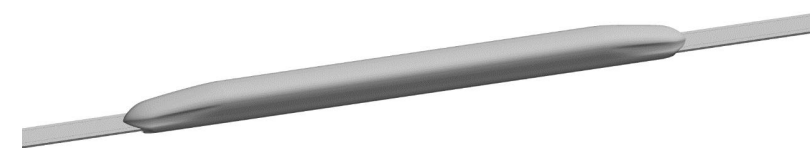

(b) 


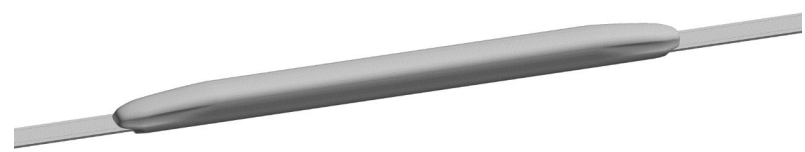

(c)

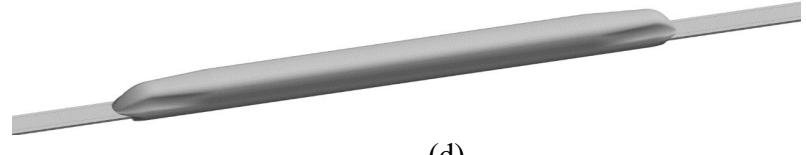

(d)

FIGURE 7: THREE-GROUP SIMPLIFIED MODEL: (a) TYPE I MODEL; (b) TYPE II MODEL; (c) TYPE III MODEL; (d) TYPE IV MODEL.

The lengths of the leading, middle and trailing cars of all the four models are $24 \mathrm{~m}, 26 \mathrm{~m}$, and $24 \mathrm{~m}$ respectively, and the total length is 72 meters. And all the maglev train models run on the track with a gap of $10 \mathrm{~mm}$. Take Type II as an example, the compartments are divided as shown in Figure 8.

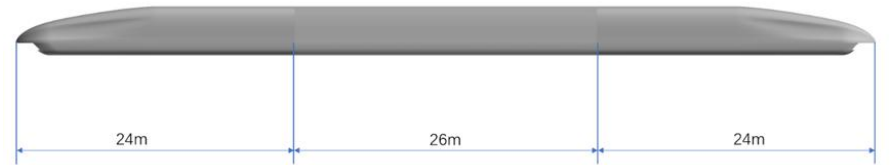

FIGURE 8: COMPARTMENT DIVISION.

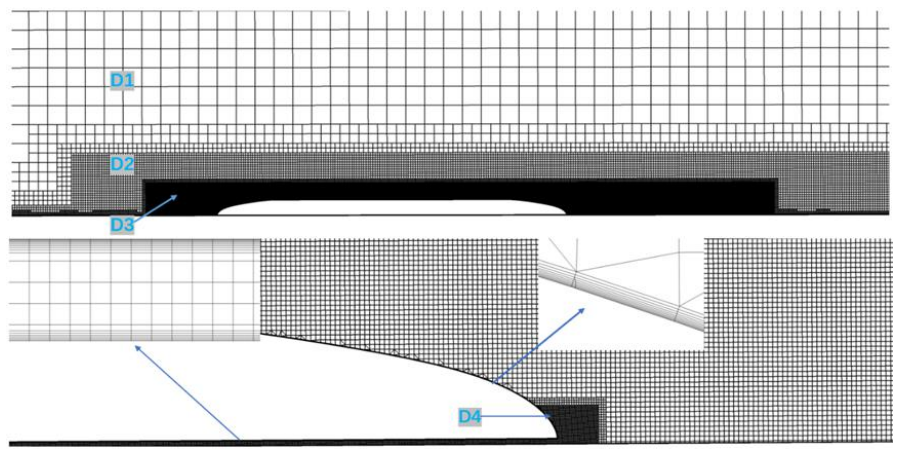

FIGURE 9: MESHING.

\subsection{Computational Grids}

The accuracy of numerical simulation is closely related to the quality of the grid, and the number of grids determines the computational cost, so the current study adopts a certain strategy for grid division. The geometry of the train is more complicated, and the gap between the train and the track is very small compared to the characteristic length of the train. And there are large and small vortices in the train wake area that need to be captured by fine grids. In this paper, the calculation area is divided into four cuboids, and each area is refined to different degrees. The area is divided into four parts: far-field area D1, near the entire train D2, near the surface of the train and wake area D3, the area containing the top of the head and tail and the gap between the train and the track D4. The grid size of the four areas of D1, D2, D3, and D4 decreases sequentially. Besides, to more accurately describe the boundary layer effect of the boundary surface, the prism grids are adopted by the commercial software Star $\mathrm{CCM}+$ and 10 layers of prism grids are generated with an increasing ratio of 1.2 and a total length of $20 \mathrm{~mm}$. In this study, the sensitivity of the grid has been analyzed, and it is not shown here due to space limitations. The grid refinement area division and the prism layer grid are shown in Figure 9. The total number of computing grids is about $4.9 * 10^{8}$.

\subsection{Computational domain and boundary conditions}

The computational domains of the four high-speed maglev trains are the same. Taking the Type II model as an example, the calculation domain is shown in Figure 10. Take the height of the maglev train as the characteristic length $\mathrm{H}$ (here $4 \mathrm{~m}$ ). In order to fully develop the flow field and reduce the influence of the boundary on the flow around the train, the length from the leading car to the boundary of the Inlet is about $20 \mathrm{H}$, and the length from the trailing car to the boundary of the Outlet is about $41.5 \mathrm{H}$. The width of the calculation domain is $40 \mathrm{H}$, and the height of the calculation domain is $20 \mathrm{H}$. Because the high-speed maglev train runs at a speed of $600 \mathrm{~km} / \mathrm{h}$, considering the compressibility of the gas, the Inlet, Outlet, Upper boundary, $\mathrm{Y}$ - boundary and $\mathrm{Y}+$ boundary are set as free flow boundary conditions, and the velocity of the airflow is $600 \mathrm{~km} / \mathrm{h}$. The calculation process also includes the simulation of ground effects. The track and the ground move relative to the train, so the boundary conditions of the ground and track are set to the same moving wall condition as the incoming flow.

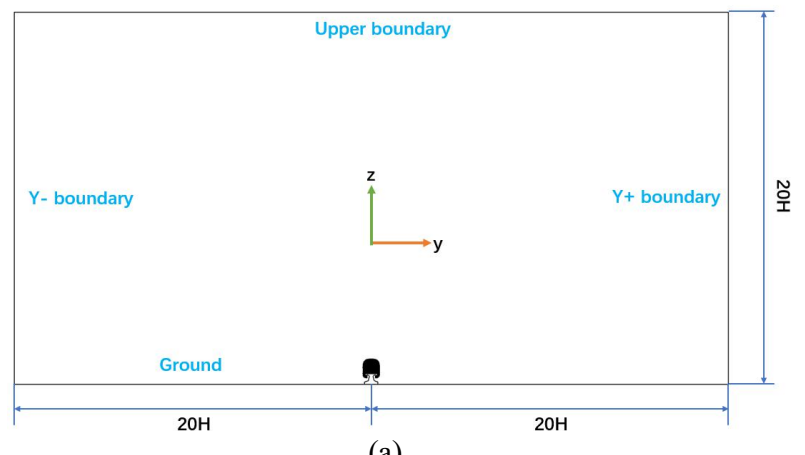

(a)

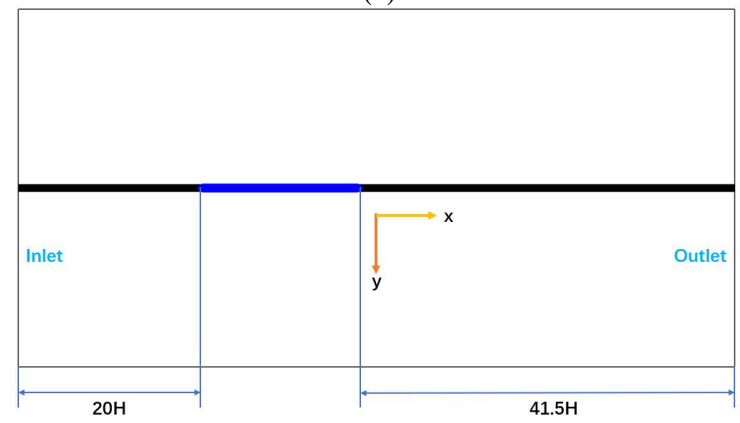

(b)

FIGURE 10: COMPUTATIONAL DOMAIN: 


\section{(a) FRONT VIEW; (b) TOP VIEW.}

\subsection{Aerodynamic coefficients} follows:

To facilitate analysis, define the aerodynamic coefficients as

$$
\begin{aligned}
\text { Aerodynamic drag coefficient: } & C_{d}=\frac{2 F_{x}}{\rho V^{2} S_{x}} \\
\text { Aerodynamic lift coefficient: } & C_{l}=\frac{2 F_{z}}{\rho V^{2} S_{x}} \\
\text { Pressure coefficient: } & C_{P}=\frac{2 \Delta p}{\rho V^{2}}
\end{aligned}
$$

In the formula, $F_{x}$ is the air resistance of the train, is the air lift of the train, $F_{z}$ is the difference between the pressure at that point and the pressure at infinity; $\rho$ is the air density, taken as $1.225 \mathrm{~kg} / \mathrm{m}^{3} ; \mathrm{V}$ is the incoming flow velocity, taken as $600 \mathrm{~km} / \mathrm{h} ; S_{x}$ is the reference area, which is the maximum cross-sectional area of the train model, $11.864 \mathrm{~m}^{2}$ for all four types of models. $\Delta p$ is the difference between the train surface pressure and the standard atmospheric pressure at infinity.

\subsection{Wind tunnel test verification}

In order to ensure the effectiveness of the numerical calculation results, this paper compares the numerical simulation results with the experimental data of wind tunnel tests. The experiment was carried out in AVIC Aerodynamics Research Institute in Harbin, Heilongjiang Province. The experimental model, as shown in Figure 11 (a), is a 1:8 scaled three-group maglev train model. The numerical simulation model, computational domain and boundary conditions are exactly the same as the experiment, and SST k- $\omega$ is selected as the turbulence model. The numerical simulation adopts the strategy of mesh refinement around the train and the wake area to capture the flow details. The boundary layer setting is similar to the calculation model in this paper. Fig. 11 (b) shows the grid division of the calculation model along the longitudinal section and the grid details of the boundary layer. The total number of numerical grids is about 31.33 million.

In order to carry out numerical verification, the resistance coefficient and lift coefficient of each compartment calculated are compared with the experimental data, and the results are shown in Table 2. It can be seen that the error of each aerodynamic coefficient of each compartment is within $3.3 \%$. The comparison results show that the numerical algorithm and network configuration can accurately predict the flow field around the train.

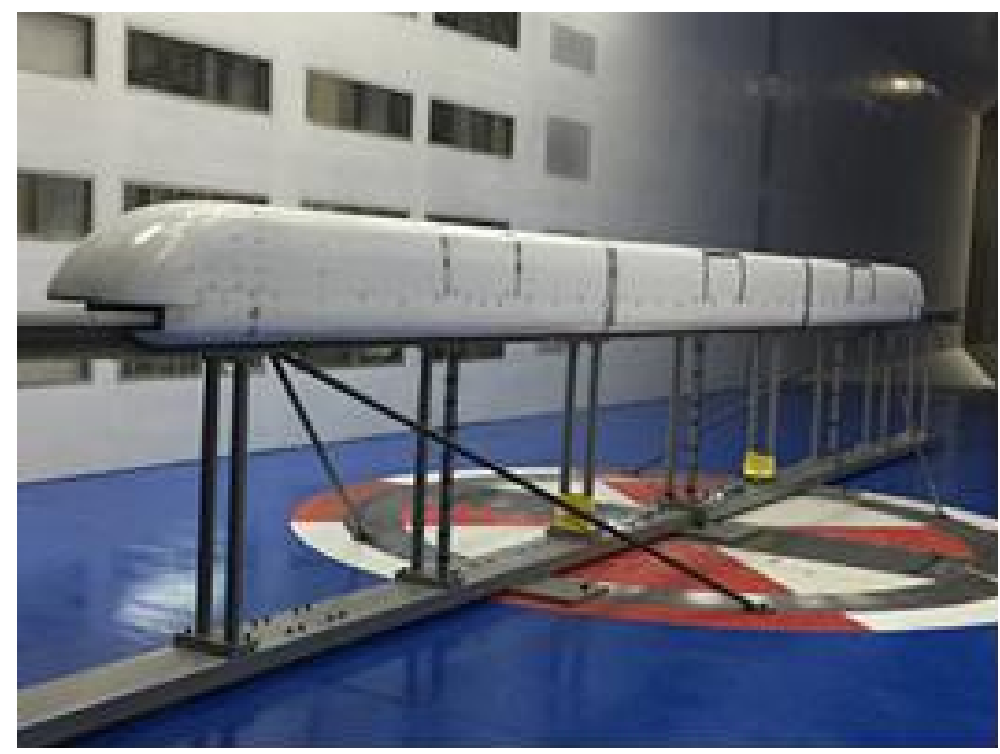

(a) Wind tunnel test model.

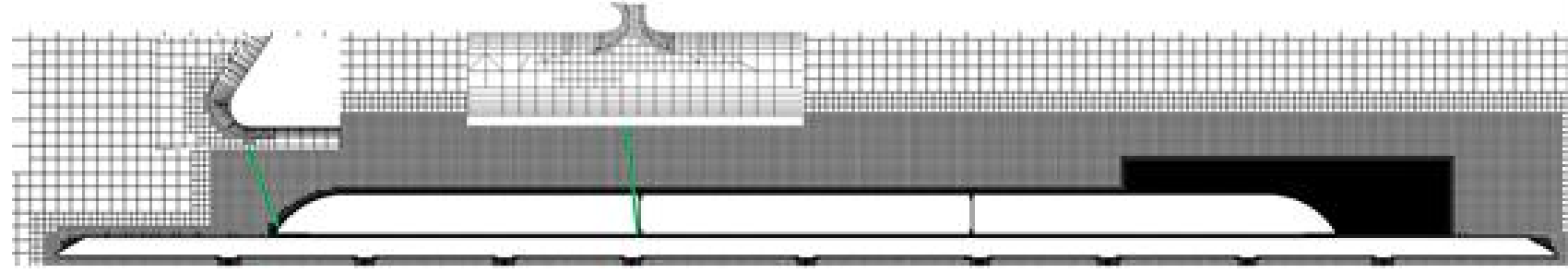

(b) Distribution of Spatial Grid.

FIGURE 11: WIND TUNNEL TEST MODEL AND SPATIAL GRID DISTRIBUTION. 
TABLE 2: COMPARISON OF CFD AND EXPERIMENTAL RESULTS.

\begin{tabular}{cccccccc}
\hline & Total- $C_{d}$ & Head- $C_{d}$ & Head- $C_{l}$ & Middle- $C_{d}$ & Middle- $C_{l}$ & Tail- $C_{d}$ & Tail-Cl \\
\hline Exp & 0.3368 & 0.0846 & 0.4169 & 0.0864 & -0.0247 & 0.1658 & $\mathbf{0 . 2 7 7 6}$ \\
CFD & 0.3279 & 0.0831 & 0.4058 & 0.0843 & -0.024 & 0.1605 & $\mathbf{0 . 2 8 3 1}$ \\
Error & $-2.64 \%$ & $-1.77 \%$ & $-2.66 \%$ & $-2.43 \%$ & $-2.83 \%$ & $-3.20 \%$ & $\mathbf{1 . 9 8 \%}$ \\
\hline
\end{tabular}

\section{RESULTS AND DISCUSSIONS}

High-speed maglev trains run on the guide rails, and aerodynamic characteristics have a huge impact on the train's operational safety, environmental performance and control system. For a high-speed maglev train with an open track, no crosswind and running smoothly, its aerodynamic characteristics are mainly composed of aerodynamic drag and aerodynamic lift. The current study simulates the aerodynamic characteristics of the train under no wind environment.

\subsection{Comparison of train aerodynamic resistance}

The study of the influence of different shapes on the resistance of the maglev train is of great significance to the design optimization work and the engineering application of the maglev train. Because the train is a slender body, the flow field and resistance of each car are very different when running in a steady state. Therefore, the current study compares each compartment and the total aerodynamic drag coefficient of four models, as shown in Table 3.

The computational results show that among the Type I, Type II and Type III models with the same slenderness ratio, the more tapered Type I has the least aerodynamic resistance, followed by Type II, and Type III, which is close to a bluff body, has the largest aerodynamic resistance. Although the nose cone shape of the Type IV model is flatter, the streamline is shorter. So, the aerodynamic resistance of Type IV is greater than Type I and Type II.

The aerodynamic resistance of a train is mainly composed of friction resistance and pressure resistance. The friction resistance is caused by air viscosity, and the pressure resistance is caused by the pressure difference between the head and tail of the train. The surface area of the four train models has little change, and the bodies are all simplified and smooth. It is considered that the friction resistance is close at the same speed. The resistance of the middle carriages of the four train models is mainly frictional resistance, which shows little change. Small changes in frictional resistance are caused by changes in the thickness of the boundary layer. Therefore, pressure resistance is the main reason for the difference in aerodynamic resistance of various trains. Since the lower surface of the train model is a flat plate parallel to the ground, there is no pressure difference in the direction of the train movement. Therefore, the pressure distribution on the upper surface of the head and tail is analyzed and compared, as shown in Figure 12.

TABLE 3: AERODYNAMIC DRAG COEFFICIENT (Cd) OF EACH PART.

\begin{tabular}{ccccc}
\hline Grouping part & Type I-Cd & Type II-Cd & Type III-Cd & Type IV-Cd \\
\hline Head & 0.0612 & 0.0657 & 0.0711 & 0.0702 \\
Middle & 0.0545 & 0.0531 & 0.0526 & 0.0532 \\
Tail & 0.0550 & 0.0602 & 0.0794 & 0.0764 \\
Whole vehicle & 0.1707 & 0.1790 & 0.2031 & 0.1998 \\
\hline
\end{tabular}

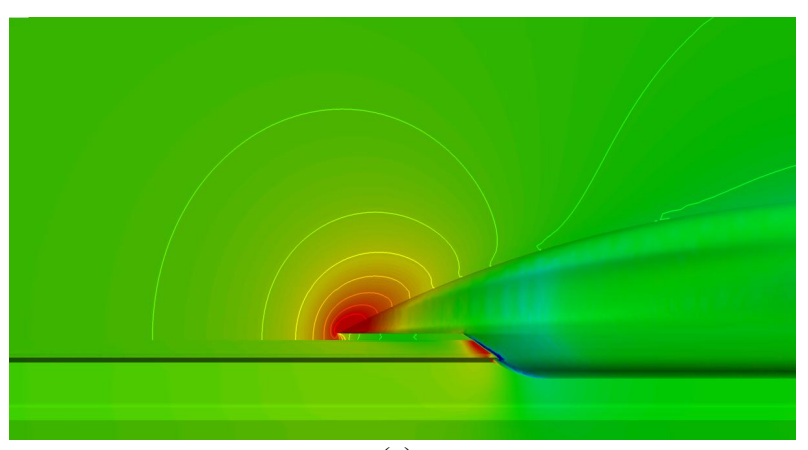

(a)

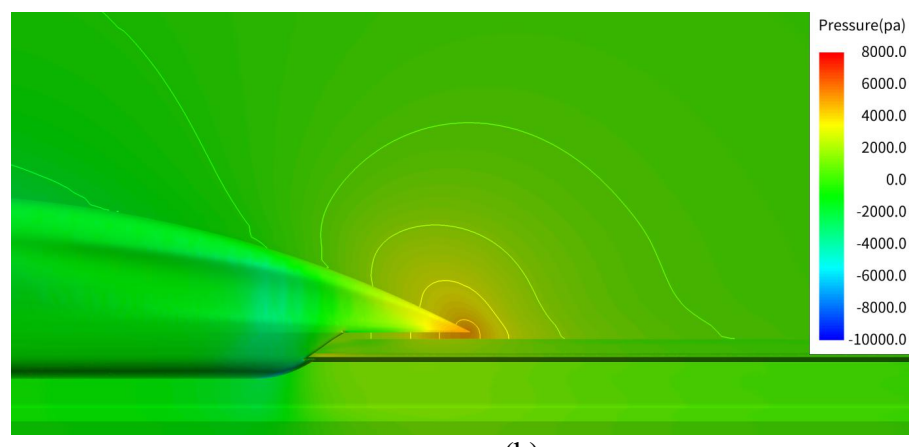

(b) 


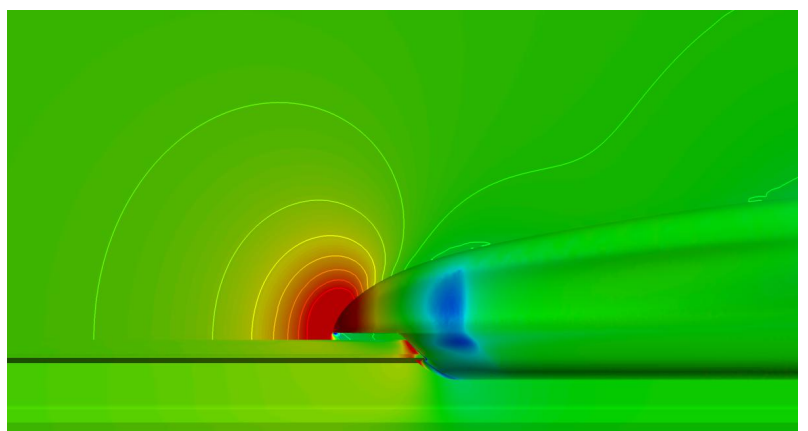

(c)

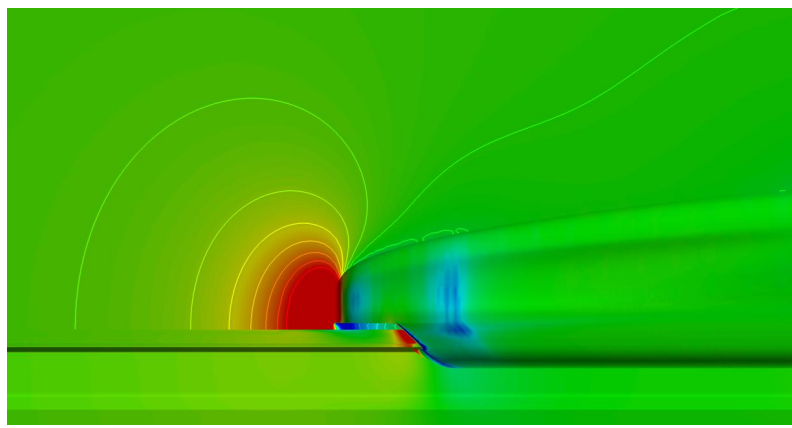

(e)

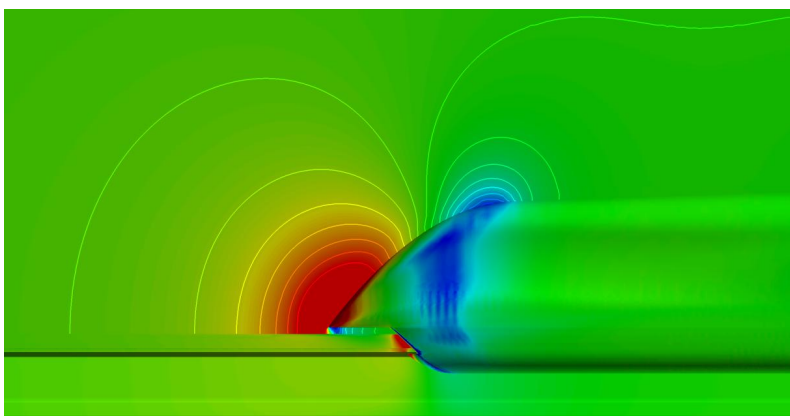

(g)

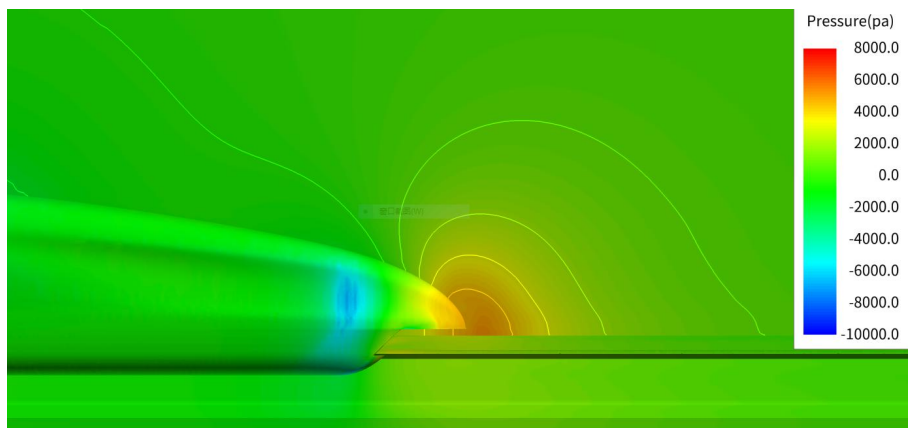

(d)

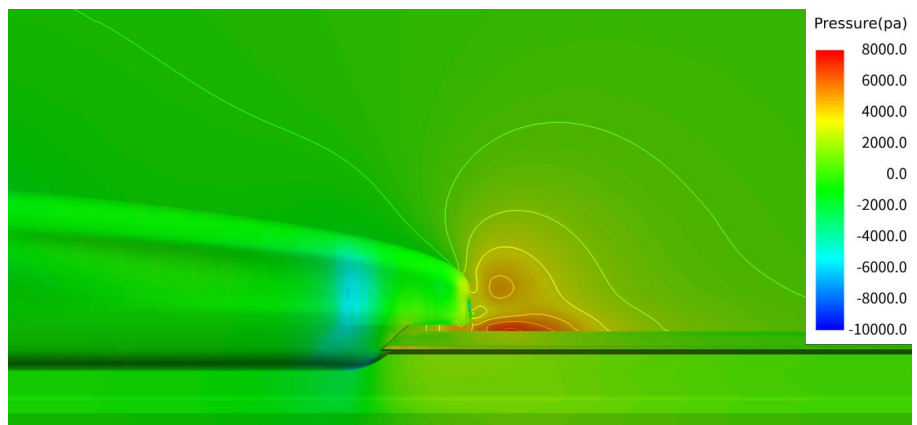

(f)

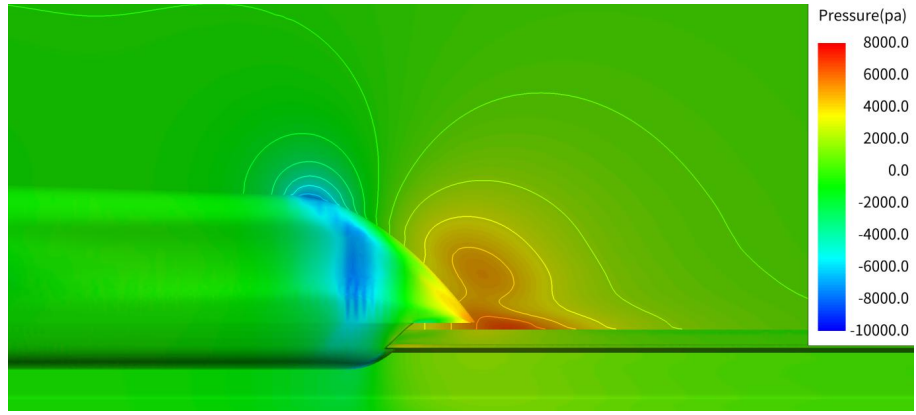

(h)

FIGURE 12: PRESSURE CONTOURS OF THE FOUR TYPES OF MODELS' HEAD AND TAIL: (a)TYPE I HEAD; (b) TYPE I TAIL; (c)TYPE II HEAD; (d) TYPE II TAIL; (e)TYPE III HEAD; (f) TYPE III TAIL; (g)TYPE IV HEAD; (h) TYPE IV TAIL.

Pressure contours of various train models are different, but the pressure distribution also has a common law. For the leading car, the incoming flow forms a stagnation point at the tip of the nose and the front end of the bottom curved surface, where the pressure is the highest, and the airflow in the surrounding area is slowed down to form a strong positive pressure zone. The velocity of the airflow increases from the strong positive pressure area upwards and to both sides, and the positive pressure gradually changes to negative pressure. The flow separation occurs in the area where the curvature of the curved surface changes greatly, forming a negative pressure area. Backward from the place where the curvature changes greatly, the negative pressure decreases. When the boundary layer structure of the flow around becomes stable, the surface pressure is maintained at a relatively weak positive pressure. For the tail of the train, as the cross section shrinks, a negative pressure is formed due to the expansion wave effect. Later, the flow separation occurs, a large number of wake vortices detach from the trailing nose, and the vortices interact with the airflow at the bottom of the train to form a weaker positive pressure zone.

The drastic air pressure changes of the chaser and the leading car are the main cause of the pressure resistance. Comparing Type I, II, and III, the curved surface of Type I does not have a large curvature change, and the horizontal and vertical profiles are the narrowest. Therefore, the air pressure change of Type I is not as drastic as the other two models, and the strength of the strong positive pressure zone and the negative pressure zone is relatively weak. Consequently, the pressure resistance of type $I$ is the smallest. Because of the shorter length of the streamlined part of the Type IV model, the curvature of the transition to the largest cross-section area changes greatly. Therefore, the flow separation produced by Type IV is more obvious, and the pressure difference resistance is relatively large. 


\subsection{Comparison of Train Aerodynamic Lift}

When the train is running at high speed, the influence of aerodynamic lift cannot be ignored. The excessive aerodynamic lift can easily cause the train suspension system to fail, and cause a collision between the bottom surface of the train and the guide rail and cause a safety accident. Therefore, the lift is a key aerodynamic characteristic of high-speed maglev trains, and reducing train aerodynamic lift can greatly improve train safety. Therefore, the current study compares each compartment and total aerodynamic lift of four train model, as shown in Table 4:

The calculation results show that among the Type I, Type II and Type III models with the same slenderness ratio, Type III has the smallest aerodynamic lift, Type I is slightly larger, and Type II is the largest. The aerodynamic lift of Type IV with a shorter streamline is greater than that of the other three models. Figure 13 shows the pressure coefficients of the upper and lower surfaces of the four high-speed maglev trains. In the figure, the abscissa -40 is the $x$ coordinate of the nose tip of the train head, and 34 is the $x$ coordinate of the nose tip of the train tail.

TABLE 4: AERODYNAMIC LIFT(Cl) OF EACH PART.

\begin{tabular}{ccccc}
\hline Grouping part & Type I-Cl & Type II-Cl & Type III-Cl & Type IV-Cl \\
\hline Head & 0.1555 & 0.2048 & 0.1925 & 0.2019 \\
Middle & 0.1076 & 0.0779 & 0.0600 & 0.0955 \\
Tail & 0.2661 & 0.2920 & 0.2512 & 0.3573 \\
Whole vehicle & 0.5302 & 0.5747 & 0.5037 & 0.6547 \\
\hline
\end{tabular}

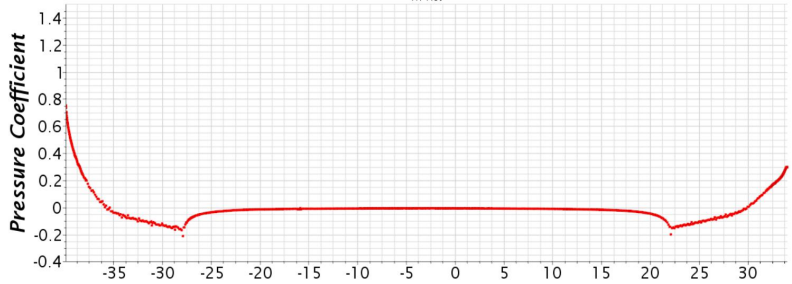

(a)

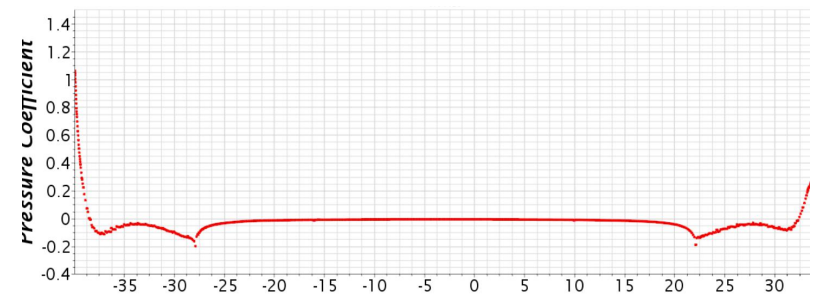

(c)

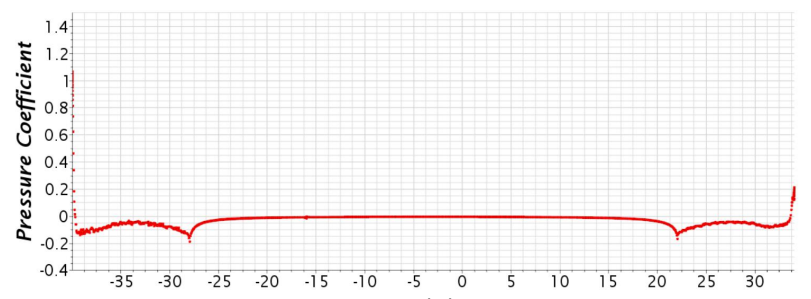

(e)

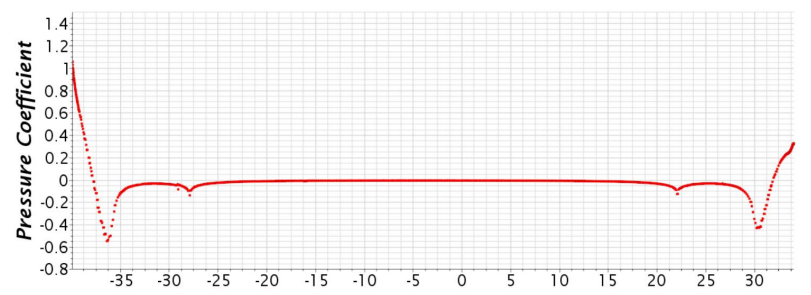

(g)

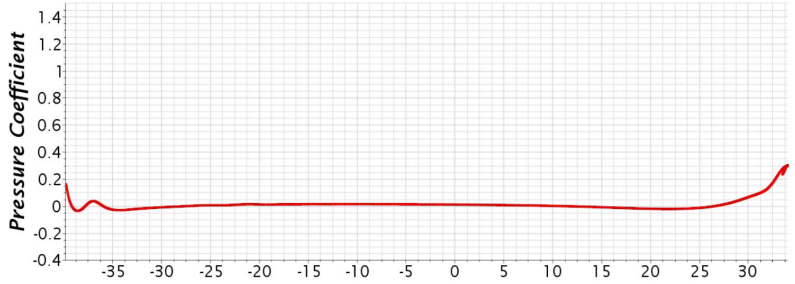

(b)

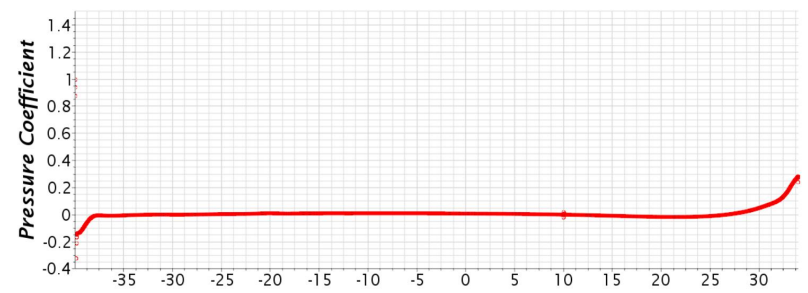

(d)

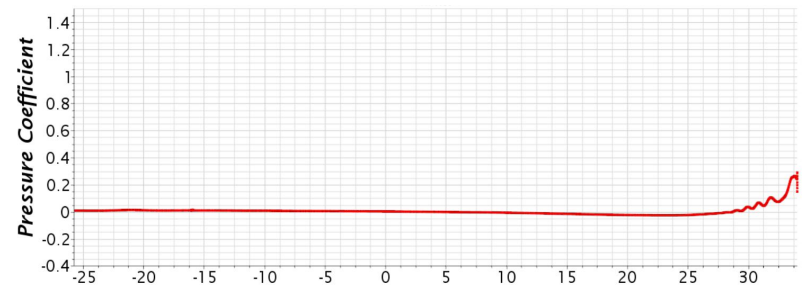

(f)

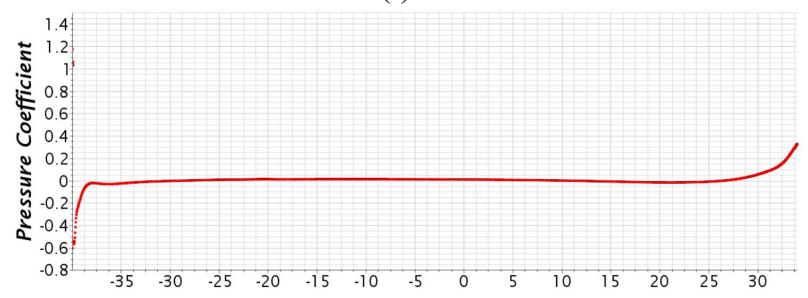

(h) 
FIGURE 13: PRESSURE DISTRIBUTION ON THE UPPER AND LOWER SURFACES OF THE FOUR TYPES MODELS: (a) TYPE I UPPER; (b) TYPE I LOWER; (c) TYPE II UPPER; (d) TYPE II LOWER; (e) TYPE III UPPER; (f) TYPE III LOWER; (g) TYPE IV UPPER; (h) TYPE IV LOWER.

When the lower surface pressure is greater than the upper surface pressure, the lift will be generated. Through the comparison of the pressure coefficient distribution on the upper and lower surfaces, it is easy to find the reason for the small lift of the type III model: the negative pressure area on the upper surface is small and the strength is low, resulting in a small pressure difference between the upper and lower surfaces of the model. Compared with Type III, the pressure difference between the upper and lower surfaces of Type II model is more obvious. There is a strong negative pressure zone on the upper surface behind the positive pressure zone at the tip of the nose, as shown in Figure (c) where $\mathrm{x}$ is $(-38,-26)$. The lower surface of this area shows weak positive pressure. There is also a strong negative pressure zone on the upper surface of the chaser, while the lower surface shows a weaker positive pressure. Therefore, the aerodynamic lift of the Type II model is greater. The same phenomenon is more obvious on the Type IV model, which in turn generates greater aerodynamic lift.

\section{COMPARISON OF WAKE CHARACTERISTICS}

The wake characteristics are an important part of the aerodynamic characteristics of trains. Since the high-speed maglev train has a unique shape, a strong interaction occurs between the trailing vortices near the tail and the guide rail. The current research numerically analyzes the difference in wake structure between trains with different topologies. When calculating the wake vortex field, the SST k- $\omega$ method is not as accurate as the unsteady method such as IDDES, but from the average point of view, the method also has certain reference significance. In this chapter, the Q-criterion is used to study the vortex structure and mechanism of different train wake regions, and the vortex propagation process is studied by TKE.

\subsection{Comparison of $Q$ criterion}

Hunt et al. [16] proposed the Q-criterion to define the vortex. The Q-criterion is the second invariant of the velocity gradient, and its expression is:

$$
Q=\frac{1}{2}\left[\left(u_{i, i}\right)^{2}-u_{i, j} u_{j, i}\right]
$$

In the expression, $u_{i}$ is the $i$-th component of the incoming velocity, and $u_{i, j}=\frac{\partial u_{i}}{\partial x_{j}}$. The Q-criterion expresses the balance between the shear strain and the vorticity. A positive Q-region means that the rotation tensor dominates the rate of the strain tensor.

Figure 14 shows the iso-surface of the Q-criterion in the wake region of the four train models when the value is 500 .

It can be observed that there are a lot of vortices existing in the wake region. The vortex structures of the four train wake regions are similar. It could be found that four vortices are propagating farther, and there are some small vortices near the nose. The small vortex is produced by the strong interaction between train and rail. There are two vortices on each side. One is generated from the side of the nose cone, which is close to the upper surface of the rail, and the other is generated from the gap between the train and the lower surface of the rail. The four vortices on both sides propagate downstream with strong intensity, which indicates that there is a strong interaction between the flow and the guide rail to provide energy for the propagation of the vortex downstream.

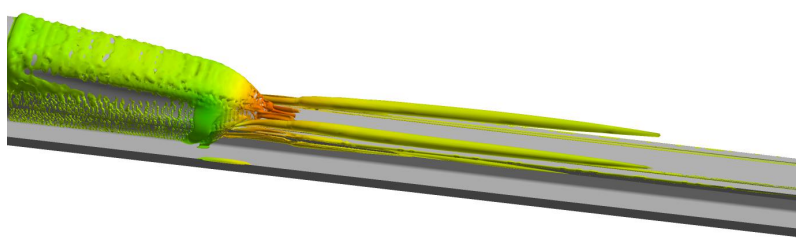

(b)

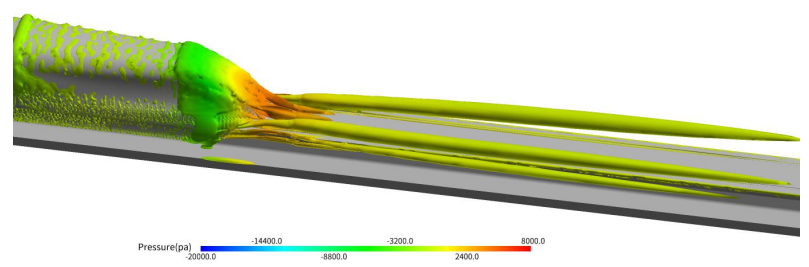

(d)

FIGURE 14: ISO-SURFACE OF Q-CRITERION IN WAKE REGION (Q=500): (a) TYPE I; (b) TYPE II; (c) TYPE III; (d) TYPE IV. 


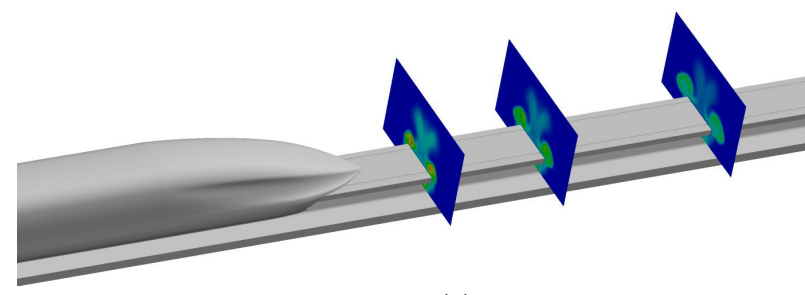

(a)

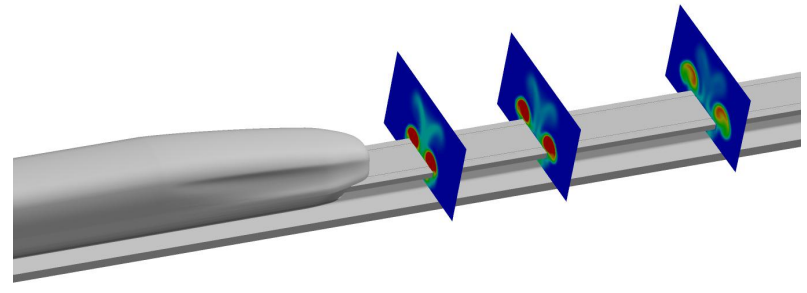

(c)

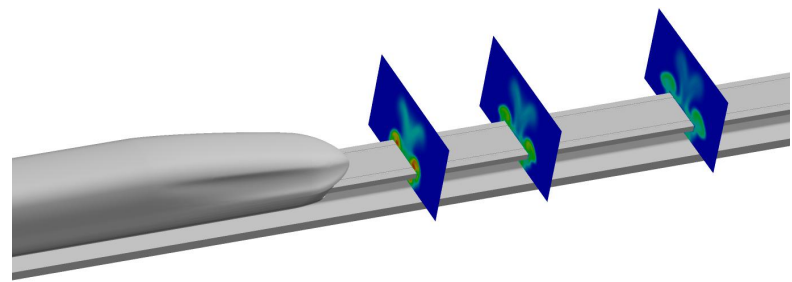

(b)

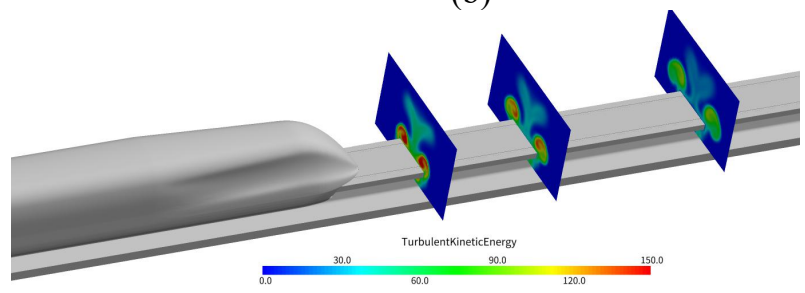

(d)

FIGURE 15: TKE CONTOUR ON THE CROSS-SECTIONS: (a) TYPE I; (b) TYPE II; (c) TYPE III; (d) TYPE IV.

Although the structure is similar, the eddy currents of the four train models also have obvious differences. Types I, II and IV produce two obvious vortex structures near the nose tip, which are the result of a strong shearing effect. The type III vortex generated near the tip of the nose and the vortex generated on the side of the nose curl each other and evolve into a vortex. Besides, for a vortex with a $\mathrm{Q}$ value of 500 , the vortex propagation distances of the four train models are also different, and the propagation distances of types I, II, III, and IV increase in turn.

\subsection{Comparison of turbulence kinetic energy}

The evolution of trailing vortices could also be studied from the view of TKE(turbulence kinetic energy). TKE is the average kinetic energy per unit mass associated with the vortex in the turbulence. Its definition is:

$$
k=\frac{1}{2}\left[\overline{\left(u^{\prime}\right)^{2}}+\overline{\left(v^{\prime}\right)^{2}}+\overline{\left(w^{\prime}\right)^{2}}\right]
$$

In the expression, $u^{\prime}, v^{\prime}$ and $w^{\prime}$ are the fluctuating velocity of the flow. TKE is an intuitive variable to represent the turbulence level of local flow, which is dissipated by viscous forces at the Kolmogorov scale.

In order to further study the dissipation of the vortex, Figure 15 shows the TKE profiles of the four train models at three different cross-sections of $\mathrm{H}, 3 \mathrm{H}$ and $6 \mathrm{H}$ in the wake, which may show the attenuation of the wake turbulence It can be seen from the figure that the main eddy currents of the four types of train models are distributed along the edge of the guide rail. The tail vortex dissipation of the four high-speed maglev trains is not obvious. The shear strain caused by the flow when the vortex flows through the guide rail provides energy input for the turbulent structure and maintains the strength of these vortices. From the perspective of turbulent kinetic energy, the vortex intensity in the wake region of Type III is the largest, followed by Type IV, and Type I is the smallest. This is consistent with the aerodynamic drag of four train models. In order to further study the dissipation of the vortex, Figure 14 shows the TKE profiles of the four train models at three different cross-sections of $\mathrm{H}, 3 \mathrm{H}$ and $6 \mathrm{H}$ in the wake, which may show the attenuation of the wake turbulence.

\section{CONCLUSION}

(1) Based on the VMF method, a parametric modeling method suitable for high-speed maglev trains is established. This method can generate train models with different topological structures.

(2) Among the four selected train models, Type I has the least aerodynamic drag. Type III has the largest aerodynamic resistance, and Type IV is second only to Type III. Analysis suggests that a flatter longitudinal profile and a narrower horizontal profile can reduce aerodynamic drag. Reducing the change of surface curvature and increasing the streamline length can also reduce aerodynamic drag.

(3) Type III train model has the smallest aerodynamic lift, followed by Type I, and Type IV the largest. By comparing the pressure distribution on the upper and lower surfaces of the four train models, it is analyzed that reducing the strength of the negative pressure zone on the upper surface can reduce the aerodynamic lift.

(4) There are four distinct vortices in the wake area of the four train models. The guide rail limits the propagation of the vortex, and the interaction between the vortex and the guide rail provides energy for the propagation of the vortex. From the perspective of turbulent kinetic energy, the vortex intensity in the wake region of Type III is the largest, followed by Type IV, and Type I the smallest.

(5) The shape design of the train pursues smaller resistance, suitable aerodynamic lift, larger train volume, and smaller wake vortex. These goals are often contradictory to each other. So the optimization design of the train shape is a game process, which 
needs to be judged based on the engineering requirements.

\section{REFERENCES}

[1] B.H. Mao, H. Rong, S.P. Jia, Application Prospect Analysis of Magnetic Levitation Technology in China, Transportation Systems Engineering and Information 01 (2008) 29-39.

[2] Raghunathan, Raghu S., H-D. Kim, and Toshiaki Setoguchi, Aerodynamics of high-speed railway train, Progress in Aerospace sciences 38.6-7 (2002): 469-514.

[3] Q.H. Wu, H.B. Yu, H.P. Li, A study on numerical simulation of aerodynamics for the maglev train. Railway Locomotive \& CAR 24.2 (2004) 18-20.

[4] D. Zhou, H.Q. Tian, Z.J. Lu, Optimization of aerodynamic shape for domestic maglev vehicle, Journal of Central South University (Science and Technology) 3 (2006) 613-617.

[5] X.F. Liang, X.Y. Shen, Lateral aerodynamic performances of maglev train when two trains meet with wind blowing, Journal of Central South University (Science and Technology) 38.4 (2007) 751-757.

[6] X.W. Shu, C.G. Gu, X.F. Liang, et al. Numerical simulation and parameterized investigation of aerodynamic drag performances of high-speed maglev trains, Journal of Traffic and Transportation Engineering 6 (2006) 6-10.

[7] P. Zhou, T. Li, C. Zhao, et al. Numerical study on the flow field characteristics of the new high-speed maglev train in open air, Journal of Zhejiang University: science A 21.5 (2020) 366-381.

[8] S. Meng, D. Zhou, S. Meng, Effect of rail gap on aerodynamic performance of maglev train, Journal of Central
South University (Science and Technology) 51(12) (2020) 3537-3545.

[9] Castonguay Patrice, Siva Nadarajah, Effect of Shape Parameterization on Aerodynamic Shape Optimization, 45th AIAA Aerospace Sciences Meeting and Exhibit (2007).

[10] Rho J H, Ku Y C, Yun S H, et al. Development of vehicle modeling function for 3-dimensional shape optimization, Mech Design 131(12) (2009) 121004-1-121004-10.

[11] Ku Y C, Kwak M H, Park H I, et al. Multi-objective optimization of high-speed train nose shape using the vehicle modeling function, 48th AIAA Aerospace Sciences Meeting (2010).

[12] Luo Xianwu, Huang Renfang, Ji Bin, 2016. Transient cavitating vortical flows around a hydrofoil using k-omega partially averaged Navier-Stokes model. Modern Physics Letters B 30 (1).

[13] Luo Xianwu, Ye Weixiang, Huang Renfang, Wang Yiwei, Du Tezhuan, Huang Chenguang, 2020. Numerical investigations of the energy performance and pressure fluctuations for a waterjet pump in a non-uniform inflow. Renewable Energy 153, 1042-1052.

[14] Zhao Xiaoli, Sun Zhenxu, A new method for numerical simulation of two trains passing by each other at the same speed, Journal of Hydrodynamics, 2010, 22(5): 697-702.

[15] Tian Li, Deng Qin, Jiye Zhang. Effect of RANS turbulence model on aerodynamic behavior of trains in crosswind, Chinese Journal of Mechanical Engineering(2019) 32:85.

[16] Hunt JCR, Wray AA, Moin P, Eddies, streams, and convergence zones in turbulent flows, Center for Turbulence Research Proceedings of the Summer Program (1988) 193-208. 